\title{
AVALIAÇÃO DE DESEMPENHO PARA 0 DESENVOLVIMENTO DO URBANISMO SUSTENTÁVEL: REVISÃO DE LITERATURA E DIRETRIZES PARA FUTURAS INVESTIGAÇÕES
}

\section{PERFORMANCEEVALUATION FOR SUSTAINABLE URBAN DEVELOPMENT: LITERATURE REVIEW AND GUIDELINES FOR FUTURE RESEARCH}

\author{
Paola Peterle Rosa do Amaral Figueiredo (UFSC); \\ Sandra Rolim Ensslin, Dra. (UFSC); \\ Adriana Marques Rossetto, Dra. (UFSC); \\ Lucas dos Santos Matos, M.Sc. (UFSC)
}

\section{Palavras Chave}

Desenvolvimento Urbano Sustentável; Avaliação de Desempenho; Revisão de Literatura; LEED ND; ProKnow-C

\section{Key Words}

Sustainable Urban Development; Performance Evaluation; Literature Review; LEED ND; ProKnow-C

\section{RESUMO}

O crescimento urbano desordenado é, hoje, um problema global. Neste contexto, a avaliação de desempenho, por meio de ferramentas para a certificação de desenvolvimento urbano sustentável, surge como uma alternativa para orientar desenvolvedores imobiliários urbanos na tomada de decisão. A pesquisa tem por objetivo abordar um fragmento da literatura referente à avaliação de desempenho para o desenvolvimento urbano sustentável, visando mapear o tema e identificar oportunidades para pesquisas futuras. Foi selecionado o instrumento metodológico ProKnow-C para a seleção e análise da literatura. O portfólio bibliográfico formado por 31 artigos apresentou os seguintes resultados: falta de reconhecimento científico sobre o tema pesquisado, desenvolvimento das ferramentas concentradas entre 2007 e 2014, e derivação das ferramentas de avaliação de desempenho de edificações. As lacunas identificadas foram: necessidade de uma rede para compartilhamento de informações, transparência sobre o peso dado a cada indicador, necessidade de avaliação de indicadores locais, dentre outros.

\section{ABSTRACT}

Disorganized urban growth is now a global problem. In this context, the evaluation of performance through tools for the certification of sustainable urban development appears as an alternative to guide urban real estate developers in decision making. The research aims to address a fragment of literature on performance evaluation for sustainable urban development, aiming at identifying opportunities and possibilities for a future research agenda. The methodological tool, called ProKnow- $C$, was chosen for selection and analysis of literature. The bibliographic portfolio consists of 31 articles with the following results: lack of scientific recognition on the researched topic, development of the tools concentrated between 2007 and 2014, and derivation of the tools of evaluation of performance of buildings. The identified gaps were: need for a network to share information, transparency on the weight given to each indicator, need to evaluate local indicators, among others. 


\section{INTRODUÇÃO}

A urbanização e a densidade populacional são sugeridas como determinantes-chave que moldarão o futuro das cidades do século (AMEEN et al., 2015). Atualmente, é um dos grandes problemas mundiais, principalmente nos países em desenvolvimento, uma vez que mais da metade da população já está vivendo em perímetros urbanizados (MADANIPOUR, 2006; HIREMATH et al., 2013; LUEDERITZA et al., 2013).

Países preocupados com o tema já começaram a elaborar estratégias para promover o desenvolvimento sustentável urbano por meio de ferramentas de análise de desempenho (ALQAHTANYA et al., 2012). Ao mesmo tempo, é preciso lembrar que o planejamento urbano, por si só, fornece uma solução parcial para o alcance do desenvolvimento urbano sustentável, pois os padrões do comportamento humano, tradições, atitudes, crenças e preconceitos podem estar além do controle, apesar dos melhores esforços dos planejadores (CHOGUILL, 2008).

A pesquisa tem por objetivo abordar um fragmento da literatura referente à avaliação de desempenho para o desenvolvimento urbano sustentável, visando mapear o tema e identificar oportunidades e possibilidades de uma agenda de pesquisa futura. Esta pesquisa justifica-se por conter, segundo Castro (1977), importância científica, originalidade e viabilidade. A sua importância deve-se ao fato de que sistemas de avaliação de desempenho, quando corretamente implantados, podem prever problemas futuros, na busca por entender e solucionar as adversidades que estão surgindo em um mundo dinâmico (NUDURUPATI et al., 2011; BITITCI et al., 2011). Entende-se por original a revisão sistemática da literatura sob o ponto de vista da avaliação de desempenho, e desenvolvimento urbano sustentável na visão da ferramenta Leadership in Energy and Environmental Design for Neighborhood Development (LEED ND). A viabilidade é dada pelo acesso ao banco de dados do Portal de Periódicos da Coordenação de Aperfeiçoamento Pessoal de Nível Superior (CAPES), da Universidade Federal de Santa Catarina, instituição governamental fomentadora da pesquisa científica no Brasil, a qual permite o acesso a bases de dados internacionais, fontes secundárias desta pesquisa (http://www.periodicos.capes.gov.br/).

\section{REFERENCIAL TEÓRICO: AVALIAÇÃO DE DESEMPENHO NO DESENVOLVIMENTO URBANO SUSTENTÁVEL}

Várias ferramentas de avaliação ambiental foram desenvolvidas para este setor visando, em um primeiro momento, à certificação de empreendimentos e, em um segundo, aumentar a escala de avaliação para áreas urbanas. Esta mudança foi consequência da constatação de que as ferramentas focadas em unidades isoladas (edificações) eram insuficientes para garantir a sustentabilidade do ambiente construído (KYRKOUA; KARTHAUSA, 2011; HAAPIO, 2012; BERARDI, 2013; AMEEN et al., 2015).

A interdependência dos indicadores acarreta novos desafios para a implementação, em particular, quando o aumento do desempenho em um indicador resulta uma correspondente diminuição do desempenho de outro indicador (AHVENNIEMI et al., 2016). Contudo, o entendimento das interconexões dos inúmeros indicadores interdependentes nas suas diferentes dimensões é a chave para a sustentabilidade urbana (AMEEN et al., 2015).

O termo Avaliação de Desempenho é visto cientificamente sobre diferentes pontos de vista. Ela é um sistema, ou seja, um conjunto de métricas, elaboradas para estarem alinhadas com os objetivos e estratégias da empresa, sendo uma ferramenta de gestão (e não somente de controle) (GHALAYINI; NOBLE, 1996), com processos flexíveis para rápida adaptação e repostas ao mercado (NUDURUPATI et al., 2011), com revisões constantes para promover melhorias, sob as dimensões financeiras, organizacionais, mercadológicas e de inovação (BOURNE et al., 2000), capaz de comunicar e influenciar comportamentos positivos das equipes (FRANCO SANTOS et al., 2007; NUDURUPATI et al., 2011). Ao final, devem levar a satisfação dos clientes e a eficiência da empresa versus o seu mercado concorrencial (NEELY; GREGORY; PLATTS, 1995), estando preparadas para um mundo em constante dinâmica e, portanto, necessitando de revisões conceituais constantes (BITITCl et al., 2012; NUDURUPATI; TEBBOUNE; HARDMAN, 2016).

As ferramentas de avaliação de desempenho urbano sustentável são chamadas, pelos pesquisadores, de métodos de avaliação da sustentabilidade urbana (USAM Urban Sustainability Assessment Methods) e de avaliação de áreas urbanas sustentáveis (NSA tool - Neighborhood Sustainable Assessment), as quais são desenvolvidas de forma hierárquica de cima para baixo, por organizações especializadas, que permitem interpretações pluralistas sobre o desenvolvimento urbano sustentável, tanto local quanto global. Foram levantadas preocupações quanto à eficácia destas ferramentas ao considerar: (a) as variações no impacto dos indicadores de sustentabilidade entre regiões e países; e (b) as variações locais no processo de tomada de decisão (HAAPIO, 2012; SHARIFI; MURAYAMA, 2015; AMEEN et al., 2015). Os pesquisadores, com uma 
proposta de adequar as interações, estão propondo novas integrações nestas ferramentas, tais como: cidadão e sociedade (BERARDI, 2013; TURCU, 2013; KOMEILY; SRINIVASAN, 2015; AHVENNIEMI et al., 2016), qualidade de vida (ALQAHTANYA et al., 2012) e cultura (AMEEN et al., 2015).

Entretanto, as fermentas elencadas nos artigos, apesar de serem denominadas de sistemas, não o são conceitualmente. A razão para isto é que sua ação acaba após a implantação, onde os decisores (desenvolvedores urbanos), que não participam da construção do modelo, somente na decisão de quais créditos/indicadores serão utilizados, deixam a gestão aos futuros ocupantes e seus aos gestores públicos. Essas divergências de conceitos ocorrem, pois, apesar de a avaliação de desempenho organizacional ser um tema bastante discutido, raramente ela é definida (NEELY; GREGORY; PLATTS, 1995; FRANCO-SANTOS et al, 2007), fazendo com que um dos desafios da área seja gerar sistemas voltados à gestão do desempenho, e não somente à sua mensuração (NEELY, 1999).

\section{METODOLOGIA DE PESQUISA}

Esta seção está subdividida em: (i) Instrumento de intervenção; (ii) Processo para Seleção do Portfólio Bibliográfico para compor o Referencial Teórico sobre Avaliação de Desempenho no Desenvolvimento Urbano Sustentável por meio da ferramenta LEED ND; e, (iii) Processo para Seleção do Portfólio Bibliográfico para compor o Referencial Teórico sobre Avaliação de Desempenho.

3.1. Instrumento de Intervenção: ProKnow-C

Esta pesquisa de abordagem qualitativa selecionou o instrumento Knowledge Development Process Constructivist (ProKnow-C), o qual orienta o pesquisador na construção do Portfólio Bibliográfico (PB). Ela tem por objetivo a identificação, análise e reflexão de fragmento da literatura sobre as ferramentas de avaliação de desempenho para o desenvolvimento urbano sustentável, através da visão da ferramenta LEED ND.

A seleção do instrumento de intervenção Knowledge Development Process - Constructivist (ProKnow-C) justifica-se por se tratar de um processo estruturado, cujo propósito é a geração de conhecimento acerca de um determinado tema, segundo as delimitações do pesquisador. Argumenta-se que o conhecimento gerado fundamenta e permite justificar as escolhas científicas do pesquisador (TASCA et al., 2010) e a identificação de oportunidades de pesquisa (VALMORBIDA, ENSSLIN, 2016, p. 126).
A aplicação do instrumento ProKnow-C é estruturada em quatro etapas: "(i) seleção do portfólio bibliográfico; (ii) bibliometria; (iii) análise sistêmica; (iv) formulação da pergunta e objetivos da pesquisa" (ENSSLIN et al., 2013, p. 9). Para o alcance do objetivo desta pesquisa, operacionalizaram-se as duas primeiras etapas do ProKnow-C, ou seja, (i) a formação de um portfólio de artigos relevantes e alinhados ao tema, e (ii) a análise bibliométrica das variáveis básicas (artigos, periódicos, autores e ferramentas de $A D$ ) e variáveis avançadas (nível do sistema/ferramenta de avaliação e ciclo de vida do sistema/ferramenta), que respondem pelo mapeamento da literatura e possibilitam a geração de conhecimento sobre o tema.

3.2. Processo para Seleção do Portfólio Bibliográfico para compor o Referencial Teórico sobre Avaliação de Desempenho no Desenvolvimento Urbano Sustentável por meio da ferramenta LEED ND

Segue Quadro 01 na próxima página. 
Quadro 01: Etapas do ProKnow-C operacionalizadas na pesquisa.

\begin{tabular}{|c|c|}
\hline \multicolumn{2}{|c|}{ Etapas do ProKnow-C } \\
\hline Definição dos eixos de pesquisa & $\begin{array}{c}\text { Eixo 1: Avaliação de Desempenho } \\
\text { Eixo 2: LEED ND } \\
\text { Eixo 3: Urbanismo Sustentável }\end{array}$ \\
\hline Seleção das Bases de Dados Consultadas & $\begin{array}{l}\text { (i) Base Wiley; (ii) Base Web of Science; (iii) Base EBSCO; } \\
\text { (iv) Base Science Direct; (v) Base SCOPUS; (vi) Base Cam- } \\
\text { bridge Core; (vii) Base Engineering Village; (viii) Base } \\
\text { ProQuest. }\end{array}$ \\
\hline Comando de Busca & $\begin{array}{c}\text { "performance measure*" or "performance evaluation*" } \\
\text { or "performance assess" or "performance appraisal" or } \\
\text { "management" or "indicator" or "critical factor" or "best } \\
\text { practices" } \\
\text { AND } \\
\text { "leed nd" or "Leadership in Energy and Environmental } \\
\text { Design for Neighborhood" or "leed-nd" or "leednd" or } \\
\text { "Neighborhood Pattern and Design" } \\
\text { AND } \\
\text { "Urbanism*" or "urban*" or "sustainable planning*" or } \\
\text { "sustainable development*" or "urbanization*" or "Sus- } \\
\text { tainable neighborhood*" or "community Structure*" or } \\
\text { "community development" or "neighborhood devel- } \\
\text { opment*" or "Urban sustainability*" or "neighborhood } \\
\text { sustainability*" or "Sustainable city*" or "smart city*" or } \\
\text { "New Urbanism*" or "sustainability*" or "urban sustain- } \\
\text { able development practices" or "urban laws" or "urban } \\
\text { policies*" or "Sustainable planning*" or "cities*" or "Sus- } \\
\text { tainable urban development*" or "process of urbaniza- } \\
\text { tion*" or "urban management*" or "urban planning*" or } \\
\text { "urban development*" }\end{array}$ \\
\hline Delimitações na Busca & $\begin{array}{l}\text { 1. Artigos publicados em periódicos científicos; } \\
\text { 2. Pesquisa por meio da disponibilidade do comando de } \\
\text { busca em: título, resumo e palavras-chave, na base de } \\
\text { periódicos da CAPES; } \\
\text { 3. Limite temporal de } 2000 \text { a } 2016 ; \\
\text { 4. Artigos em idioma inglês e português. }\end{array}$ \\
\hline No de Artigos do Banco de Dados Brutos & 2.422 artigos \\
\hline $\begin{array}{l}\text { Filtragem quanto à redundância, capítulos de livros, } \\
\text { artigos e outros }\end{array}$ & 487 artigos excluídos \\
\hline Filtragem pelo título sem alinhamento & 1.846 artigos excluídos \\
\hline $\begin{array}{l}\text { Filtragem pelo Reconhecimento Científico no Google } \\
\text { Scholar }\end{array}$ & 39 artigos excluídos \\
\hline Filtragem pela Leitura do Resumo & 30 artigos foram excluídos \\
\hline $\begin{array}{l}\text { Análise dos artigos sem reconhecimento científico em } \\
\text { relação a serem recentes ( } 2015 \text { e 2016) e por terem } \\
\text { autores selecionados anteriormente }\end{array}$ & 5 artigos foram selecionados \\
\hline Disponibilidade Integral e gratuita dos artigos & Nenhum artigo foi excluído \\
\hline Teste de Representatividade & 4 artigos foram selecionados \\
\hline $\begin{array}{l}\text { Análise do Referencial Bibliográfico dos Artigos selecio- } \\
\text { nados no Teste de Representatividade }\end{array}$ & 2 artigos foram selecionados \\
\hline $\begin{array}{l}\text { Artigos selecionados por meio do intrumento ProK- } \\
\text { now-C }\end{array}$ & 31 artigos \\
\hline
\end{tabular}


A partir do ProKnow-C foi selecionado o Portfólio Bibliográfico, que informará a redação da seção de referencial teórico, composto por 31 artigos internacionais sobre o tema desenvolvimento urbano sustentável, na visão da avaliação de desempenho, tendo como base a certificação LEED ND, respondente ao fragmento da literatura apresentado no Quadro 02.

AHVENNIEMI, H. (et al). What are the differences between sustainable and smart cities? Journal Cities. Volume 60, Part A. Páginas 234-245. 2017.

ALBINO, Vito; BERARDI, Umberto; DANGELICO, Rosa Maria. Smart cities: Definitions, dimensions, performance, and initiatives. Journal of Urban Technology, v. 22, n. 1, p. 3-21, 2015

ALQAHTANY, Ali; REZGUI, Yacine; LI, Haijiang. A proposed model for sustainable urban planning development for environmentally friendly communities. Architectural Engineering and Design Management, v. 9, n. 3, p. 176-194, 2013

AMEEN, Raed Fawzi Mohammed; MOURSHED, Monjur; LI, Haijiang. A critical review of environmental assessment tools for sustainable urban design. Environmental Impact Assessment Review, v. 55, p. 110-125, 2015

BEILER, Michelle Oswald; WAKSMUNSKI, Emily. Measuring the Sustainability of Shared-Use Paths: Development of the GreenPaths Rating System. Journal of Transportation Engineering, v. 141, n. 11, p. 04015026, 2015

BERARDI, Umberto. Sustainability assessment of urban communities through rating systems. Environment, development and sustainability, v. 15, n. 6, p. 1573-1591, 2013

BUGLIARELLO, George. Urban sustainability: Dilemmas, challenges and paradigms. Technology in Society, v. 28, n. 1, p. 19-26, 2006

CAMPBELL, Heather. The darker side of local communities: is this the real world of planning? Planning Theory \& Practice, v. 6, n. 4, p. 517-519, 2005

CHOGUILL, Charles L. Developing sustainable neighbourhoods. Habitat International, v. 32, n. 1, p. 41-48, 2008

CLOUTIER, Scott; JAMBECK, Jenna; SCOTT, Norman. The Sustainable Neighborhoods for Happiness Index (SNHI): A metric for assessing a community's sustainability and potential influence on happiness. Ecological Indicators, v. 40, p. $147-152,2014$

DLOUHÁ, Jana et al. Learning for sustainable development in regional networks. Journal of Cleaner Production, v. 49 , p. $1-4,2013$

FORREST, Nigel; WIEK, Arnim. Learning from success - toward evidence-informed sustainability transitions in communities. Environmental Innovation and Societal Transitions, v. 12, p. 66-88, 2014

GARDE, Ajay. Sustainable by design? Insights from US LEED-ND pilot projects. Journal of the American Planning Association, v. 75, n. 4, p. 424-440, 2009

GREENWOOD, Ted. Bridging the divide between community indicators and government performance measurement. National Civic Review, v. 97, n. 1, p. 55-59, 2008

HAAPIO, Appu. Towards sustainable urban communities. Environmental Impact Assessment Review, v. 32, n. 1, p. 165-169, 2012

HIREMATH, Rahul B. et al. Indicator-based urban sustainability-a review. Energy for sustainable development, v. 17, n. 6, p. 555-563, 2013

KNACK, R. E. LEED-ND: What the skeptics say. Planning, v. 76, p. 18-21, 2010

KOMEILY, Ali; SRINIVASAN, Ravi S. A need for balanced approach to neighborhood sustainability assessments: A 
critical review and analysis. Sustainable Cities and Society, v. 18, p. 32-43, 2015

KOMEILY, Ali; SRINIVASAN, Ravi S. What is Neighborhood Context and Why does it Matter in Sustainability Assessment? Procedia Engineering, v. 145, p. 876-883, 2016

KYRKOU, Dimitra; KARTHAUS, Roland. Urban sustainability standards: predetermined checklists or adaptable frameworks? Procedia Engineering, v. 21, p. 204-211, 2011

LUEDERITZ, Christopher; LANG, Daniel J.; VON WEHRDEN, Henrik. A systematic review of guiding principles for sustainable urban neighborhood development. Landscape and Urban Planning, v. 118, p. 40-52, 2013

MADANIPOUR, Ali. Roles and challenges of urban design. Journal of Urban Design, v. 11, n. 2, p. 173-193, 2006

NEACȘU, Marius Cristian. The city image and the local public administration: a working tool in urban planning. Transylvanian Review of Administrative Sciences, v. 5, n. 27, p. 172-188, 2009

OLDENHUIZING, Jan; DE KRAKER, Joop; VALKERING, Pieter. Design of a quality-of-life monitor to promote learning in a multi-actor network for sustainable urban development. Journal of Cleaner Production, v. 49, p. 74-84, 2013

REITH, András; OROVA, Melinda. Do green neighbourhood ratings cover sustainability?. Ecological Indicators, v. 48, p. $660-672,2015$

ROHE, William M. From local to global: One hundred years of neighborhood planning. Journal of the American Planning Association, v. 75, n. 2, p. 209-230, 2009

SHARIFI, Ayyoob; MURAYAMA, Akito. A critical review of seven selected neighborhood sustainability assessment tools. Environmental Impact Assessment Review, v. 38, p. 73-87, 2013

SRIVASTAVA, Malini. Framework to Assess City-scale Sustainability. Procedia Engineering, v. 145, p. 1440-1447, 2016

TALEN, Emily et al. LEED-ND as an urban metric. Landscape and Urban Planning, v. 119, p. 20-34, 2013

TURCU, Catalina. Re-thinking sustainability indicators: local perspectives of urban sustainability. Journal of Environmental Planning and Management, v. 56, n. 5, p. 695-719, 2013

WANGEL, Josefin et al. Certification systems for sustainable neighbourhoods: What do they really certify?. Environmental impact assessment review, v. 56, p. 200-213, 2016.

\subsection{Processo para Seleção do Portfólio Bibliográfico para compor o Referencial Teórico sobre Avaliação de Desempenho}

Da mesma forma que o primeiro Portfólio Bibliográfico (descrito na seção 3.2), foram seguidas as etapas propostas pelo instrumento de intervenção ProKnow- $C$ para a seleção dos artigos teóricos que subsidiarão a elaboração do referencial teórico para o fragmento da literatura Avaliação de Desempenho no contexto organizacional. Desta forma, o procedimento realizado para a Seleção do PB não será detalhado.

Inicialmente, foram definidos os eixos de pesquisa: o Eixo 1 - Avaliação de Desempenho, é representado pelas seguintes palavras-chave, na língua inglesa: performance management, performance measurement, performance evaluation, performance assessment e performance appraisal; já o Eixo 2 - Teórico, é representado pelas seguintes palavras-chave, na língua inglesa: theoretical, review, systematic, systemic, state of art, state of the art, research, agenda, revisiting, revision, conceptual e revise.

Para a busca dos artigos elegeu-se, a partir do Portal de Periódicos da CAPES as bases de dados: EBSCO Academic Search Premier; Emerald Group Publishing; ProQuest; Web of Knowledge; Scopus; e Science Direct. Considerando as mesmas delimitações e procedimentos utilizados na seção 3.2, ao final, obtiveram-se 08 artigos que respondem pelo fragmento da literatura que aborda a evolução teórica da área de conhecimento Avaliação de Desempenho, apresentados no Quadro 03. 
Quadro 03: Portfólio Bibliográfico do tema Avaliação de Desempenho

BITITCI, U.; GARENGO, P.; DÖRFLER, V.; NUDURUPATI, S.. Performance measurement: Challenges for tomorrow*. International Journal of Management Reviews, v. 14, n. 3, p. 305-327, 2012.

BOURNE, M.; MILLS, J.; WILCOX, M.; NEELY, A.; PLATTS, K. Designing, implementing and updating performance measurement systems. International Journal of Operations and Production Management, v.20, n.7, p. 754-771, 2000.

GHALAYINI, A. M.; NOBLE, J. S. The changing basis of performance measurement. International Journal of Operations and Production Management, v. 16, n. 8, p. 63-80, 1996.

FRANCO-SANTOS, M.; KENNERLEY, M.; MICHELI, P.; MARTINEZ, V.; MASON,S.; MARR, B.; GRAY,D.; NEELY. A. Towards a Definition of a Business Performance Measurement System. International Journal of Operations and Production Management, v. 27, n. 8, p. 784-801, 2007.

NEELY, A. The performance measurement revolution: why now and what next? International Journal of Operations \& Production Management, v. 19, n. 2, p. 205-228, 1999.

NEELY, A.; GREGORY, M.; PLATTS, K. Performance measurement system design - A literature review and research agenda. International Journal of Operations \& Production Management, v.15, n.4, p.80-116, 1995.

NUDURUPATI, S.S.; TEBBOUNE, S.; HARDMAN, J. Contemporary performance measurement and management (PMM) in digital economies, Production Planning \& Control, v.27, n.3, p. 226-235, 2016.

NUDURUPATI, S.S.; BITICI, U.S.; KUMAR, V.; CHAN, F.T.S. State of the art literature review on performance measurement. Computers \& Industrial Engineering, v. 60, n. 2, p. 279-290, 2011.

Fonte: Autores

\section{RESULTADOS: ANÁLISE BIBLIOMÉTRICA E OPORTUNIDADES DE PESQUISA}

Esta seção está subdividida em: (i) Bibliometria: Variáveis Básicas; (ii) Bibliometria: Variáveis Avançadas; e, (iii) Oportunidades de Pesquisa.

\subsection{Bibliometria: Variáveis Básicas}

Por meio do cruzamento do número de ocorrência da variável no PB com o número de ocorrência nas referências desse PB (gráficos de dispersão), identificaram-se os seguintes destaques nas variáveis Artigos, Periódicos e Autores:

a) Artigos: Número de referências do portfólio bibliográfico versus número de citações no Google Scholar, com destaque para: A critical review of seven selected neighborhood sustainability assessment tools (SHARIFI; MURAYAMA, 2013), Towards sustainable urban communities (HAAPIO, 2012) e Sustainable by Design?Insights From U.S. LEED ND Pilot Projects (GARDE, 2009).

b) Periódicos: Número de artigos do Portfólio Bibliográfico no Periódico versus número de artigos das Referências Bibliográficas no Portfólio Bibliográfico, com destaque para Journal of the
American Planning Association Landscape and Urban Planning, Journal of Cleaner Production, Journal of Environmental Impact Assessment Review e Procedia Engineering.

c) Autores: Número de artigos do Portfólio Bibliográfico no Portfólio Bibliográfico versus número de artigos do autor nas Referências Bibliográficas, com destaque para Komeily, A., Srinivasan, R. S., Angelidou, M., Haapio, A., Hollands, R. e Berardi, U.

Em relação às ferramentas mais utilizadas nos 31 artigos (11 são teóricos), foram: LEED ND, BREEAM Communities, CASBEE for Urban Development, DGNB UD e Pearl Community Rating System.

Com base na análise das variáveis básicas, verificou-se a falta de reconhecimento científico, e a análise temporal demonstra que as ferramentas sobre o desenvolvimento urbano sustentável foram elaboradas em intervalos próximos entre 2007 e 2014, ratificando que o tema é relativamente recente em nível global. Além disso, os instrumentos de avaliação têm recebido grande atenção da comunidade científica devido à quantidade de métodos de avaliação publicados em um curto período (AMEEN et al., 2015).

Os métodos de avaliação de sustentabilidade para desenvolvimento urbano nos últimos anos tornaram-se um 
campo de pesquisa ativa (AMEEN et al., 2015; LUEDERITZ; LANG; VON WEHRDEN, 2013), especialmente com a introdução das ferramentas CASBEE for Urban Development, BREEAM Communities e LEED ND.

\subsection{Bibliometria: Variáveis Avançadas}

As variáveis avançadas foram analisadas a partir da ferramenta de avaliação de desempenho apresentada no estudo em termos da análise: (i) do nível da sistema/ferramenta de avaliação (Nível de Medidas individuais de desempenho, e/ou Nível de Conjunto de medidas de desempenho, e/ou Relação entre o Sistema de AD e ou Ambiente) com base na proposta de Neely, Gregory e Platts (1995, p.80-116) no artigo Performance measurement system design - A literature review and research agenda; e (ii) ciclo de vida do sistema/ferramenta (Desenho/design e/ou Implementação e/ou Uso/revisão do sistema) com base na proposta de Bourne et al. (2011, p. 279-290) no artigo Designing, implementing and updating performance measurement systems.

Não existe visão, em nenhum dos artigos, sobre um sistema de avaliação de desempenho.

As ferramentas já tiveram seu desenho construído por entidades, portanto não são passíveis de serem adaptadas ou construídas, uma das grandes críticas das ferramentas pelos autores. Mesmo ferramentas mais genéricas, como o LEED ND, são criticadas por essa limitação.

a) As ferramentas de AD pesquisadas nos artigos científicos avaliadas possuem as seguintes características:

a.1) Em relação à implementação, possuem coleta de dados, mensuração e diagnóstico, que são necessários no processo de certificação, mas não oferecem sistemas de gestão. Em relação à comparação, apesar de vários artigos, em sua maioria, buscarem avaliar as ferramentas de avaliação, estas comparações são teóricas, não havendo comparação real de duas ferramentas ou mais em um mesmo projeto.

a.2) Não existe uso ou revisão do sistema nas ferramentas avaliadas.

b) Cinco artigos apresentaram novas propostas de ferramentas, ressaltando que:

b.1) No artigo A proposed model for sustainable urban planning development for environmentally friendly communities, Alqahtany, Rezgui e Li (2013) informam que pretendem desenvolver um novo quadro que possa ser o primeiro passo na criação de um modelo de desenvolvimento de planejamento urbano sustentável eficaz e aplicável em todo o mundo; b.2) No artigo Design of a Quality-of-Life monitor to promote learning in a multiactor network for sustainable urban Development, Oldenhuizing, DE Kraker e Valkering (2013) propõem a criação de um software que servirá como ferramenta, baseados em princípios genéricos e, portanto, não definidos na pesquisa;

b.3) No artigo The Sustainable Neighborhoods for Happiness Index (SNHI): A metric for assessing a community's sustainability and potential influence on happiness Cloutier, Jambeck e Scott (2014) propõem uma abordagem abrangente para indexar a relação entre o desenvolvimento urbano sustentável de uma vizinhança e a felicidade;

b.4) Segundo o artigo A critical review of environmental assessment tools for sustainable urban design, Ameen, Mourshed e Li (2015) criaram duas listas de indicadores que sugerem o uso pelos profissionais, ou mesmo entidades públicas, como uma ferramenta;

b.5) No artigo Measuring the sustainability of shared-use paths: Development of the GreenPaths rating system, Beiler e Waksmunski (2015) buscam criar um desenho de AD com foco em GreenPaths (pedestre e ciclismo).

\subsection{Oportunidades de Pesquisa}

Os planejadores precisam desenvolver abordagens locais apropriadas para incentivar e promover projetos de desenvolvimento urbano sustentáveis, bem como entender seus pontos fortes e limitações com base em uma correta leitura da cidade.

As ferramentas para o desenvolvimento urbano sustentável não analisam o maior problema de uma cidade, ou fragmento dela, que é a gestão, uma vez que a cidade é uma construção dinâmica, e nem possuem visão de futuro.

Portanto, seguem as oportunidades de pesquisa identificadas:

a) Teóricas/Pesquisadores/Acadêmicos

i. Quais informações são necessárias para uma leitura da cidade?

ii. Identificar quais ferramentas de avaliação apresentam interesse para investidores, incorporadores, governos e comunidade.

iii. Quais são os problemas, desafios e benefícios das ferramentas já aplicadas? O que é local e quais podem ser aplicadas em outras regiões ou países?

iv. Como deve ser feita a comunicação para os atores, 
uma vez que se tenha conseguido a certificação através das ferramentas de AD?

v. Quais são os principais indicadores para que um projeto urbano seja considerado sustentável?

b) Praticantes/Usuários do tema

i. Quais são os problemas, desafios e benefícios das ferramentas já aplicadas e os contextos?

ii. Quais são os principais indicadores escolhidos? Como são avaliados e monitorados?

iii. Quais foram as mudanças ocorridas na Fase de Projeto e na Fase de Implantação?

iv. Quais estratégias de comunicação são realizadas (comunidade impactada, compradores, investidores e etc.)?

\section{CONCLUSÕES}

Esta pesquisa teve por objeto abordar um fragmento da literatura referente à avaliação de desempenho para o desenvolvimento urbano sustentável, visando à geração de conhecimento para mapear a literatura do tema e identificar oportunidades e possibilidades de uma agenda de pesquisa futura dentro desta área de conhecimento. Portanto, esta pesquisa selecionou o instrumento ProKnow-C para a seleção da literatura e, consequentemente, sua análise textual.

O referenciado objetivo foi alcançado por meio da operacionalização do instrumento ProKnow- $C$, que selecionou um portfólio bibliográfico de 31 artigos, onde foram realizadas as análises básicas e avançadas, que permitiram a geração de conhecimento para que as autoras tivessem condições de apontar oportunidades de pesquisa para evolução da área.

A análise bibliométrica informou que:

a. O artigo científico de maior relevância no PB e nas referências do PB é A critical review of seven selected neighborhood sustainability assessment tools, dos autores Ayyoob Sharifi e Akito Murayama, publicado em 2013. O objetivo foi o de avaliar a performance das ferramentas de avaliação de desenvolvimento no desenvolvimento urbano sustentável através da comparação de sete ferramentas. Isto denota a preocupação acadêmica focada em entender o interesse do mercado, uma vez que o tempo de concepção, elaboração de projeto e construção são de médio e longo prazos;

b. Dos 65 autores presentes no PB, distribuídos em 15 países, nenhum possui trajetória de pesquisa no tema investigado; c. Os métodos de avaliação de sustentabilidade para desenvolvimento urbano, nos últimos anos, tornaram-se um campo de pesquisa ativa, especialmente com a introdução das ferramentas CASBEE-UD, BREEAM Communities e LEED ND, ao expandirem o escopo de $A D$ de empreendimentos para uma escala de desenvolvimento urbano (AMEEN et al., 2015);

d. Confirmou-se o alinhamento das palavras-chave;

e. A ferramenta mais utilizada do PB é a LEED ND, justificada pelos autores devido ao grande número de projetos já certificados;

f. Não existe reflexão sobre o conceito de sistema de avaliação. Muitos autores confundem o conceito de sistema com o conceito de ferramenta;

g. O foco está na avaliação da ferramenta e não na problemática urbana do crescimento desordenado, através de uma definição que busque o consenso e os indicares essenciais para avaliar o desempenho de um projeto urbano sustentável;

h. Consenso dos autores de que o foco, hoje, está nas questões ambientais, dando pesos diferentes para outras dimensões da sustentabilidade;

i. Consenso entre os autores de que não existe ferramenta que possibilita uma adaptação a todos os países, sendo necessária a reflexão sobre o local onde está sendo inserido o projeto.

Para que exista um avanço no Brasil sobre o tema do desenvolvimento urbano sustentável deverá haver o fortalecimento da cultura cadastral, objetivando uma transformação para uma visão de Sistema de Gestão Municipal buscando minimizar a problemática urbana do crescimento desordenado.

\section{REFERÊNCIAS}

AHVENNIEMI, Hannele; Huovila, Aapo; Pinto-Seppä, Isabel; Airaksinen. What are the differences between sustainable and smart cities?. Cities, v. 60, p. 234-245, 2017.

ALBINO, Vito; BERARDI, Umberto; DANGELICO, Rosa Maria. Smart cities: Definitions, dimensions, performance, and initiatives. Journal of Urban Technology, $v$. 22, n. 1, p. 3-21, 2015.

ALQAHTANY, Ali; REZGUI, Yacine; LI, Haijiang. A proposed model for sustainable urban planning development for environmentally friendly communities. Architectural Engineering and Design Management, v. 9, n. 3, p. 176-194, 2013. 
AMEEN, Raed Fawzi Mohammed; MOURSHED, Monjur; LI, Haijiang. A critical review of environmental assessment tools for sustainable urban design. Environmental Impact Assessment Review, v. 55, p. 110-125, 2015.

BEILER, Michelle Oswald; WAKSMUNSKI, Emily. Measuring the Sustainability of Shared-Use Paths: Development of the GreenPaths Rating System. Journal of Transportation Engineering, v. 141, n. 11, p. 04015026, 2015.

BERARDI, Umberto. Sustainability assessment of urban communities through rating systems. Environment, development and sustainability, v. 15, n. 6, p. 1573-1591, 2013.

BITITCI, U. S.; GARENGO, P.; DORFLER, V.; NUDURUPATI, S. S. Performance Measurement: Challenges for Tomorrow. International Journal of Management Reviews, v. 14, n. 3, p. 305-327, Sep, 2012.

BOURNE, M.; MILLS, J.; WILCOX, M.; NEELY, A.; PLATTS, $\mathrm{K}$. Designing, implementing and updating performance measurement systems. International Journal of Operations \& Production Management, v. 20, n. 7, p. 754$771,2000$.

BUGLIARELLO, George. Urban sustainability: Dilemmas, challenges and paradigms. Technology in Society, v. 28, n. 1, p. 19-26, 2006.

CAMPBELL, Heather. The darker side of local communities: is this the real world of planning?. 2005.

CARDOSO, T. L.; ENSSLIN, S. R.; ENSSLIN, L.; RIPOLLFELIU, V. M.; DUTRA, A. Reflexões para avanço na área de Avaliação e Gestão do Desempenho das Universidades: uma análise da literatura científica. In: Anais do Seminários em Administração (XVIII SEMEAD) São Paulo (SP). 04 a 06 de novembro de 2015.

CHOGUILL, Charles L. Developing sustainable neighbourhoods. Habitat International, v. 32, n. 1, p. 41-48, 2008.

CLOUTIER, Scott; JAMBECK, Jenna; SCOTT, Norman. The Sustainable Neighborhoods for Happiness Index (SNHI): A metric for assessing a community's sustainability and potential influence on happiness. Ecological Indicators, v. 40, p. 147-152, 2014.

DLOUHÁ, Jana; BARTON, Andrew; HUISINGH, Donald; ADOMSSENT, Maik. Learning for sustainable development in regional networks. Journal of Cleaner Production, v. 49, p. 1-4, 2013.

DUTRA, A.; RIPOLL-FELIU, V.M.; FILLOL, A. G.; ENSSLIN, S. R.; ENSSLIN, L. The construction of knowledge from the scientific literature about the theme seaport performance evaluation. International Journal of Productivity and Performance Management, v. 64, iss 2, p. 243 - 269, 2015.

ENSSLIN, S. R.; ENSSLIN, L.; IMLAU, J. M.; CHAVES, L. C. Processo de mapeamento das publicações científicas de um tema: portfólio bibliográfico e análise bibliométrica sobre avaliação de desempenho de cooperativas de produção agropecuária. Revista de Economia e Sociologia Rural (Impresso), v. 52, p. 587-608, 2014.

FORREST, Nigel; WIEK, Arnim. Learning from successtoward evidence-informed sustainability transitions in communities. Environmental Innovation and Societal Transitions, v. 12, p. 66-88, 2014.

FRANCO-SANTOS, M.; KENNERLEY, M.; MICHELI, P.; MARTINEZ, V.; MASON, S.; MARR, B.; GRAY, D.; NEELY, Andrew. Towards a definition of a business performance measurement system. International Journal of Operations \& Production Management, v. 27, n. 8, p. 784801, 2007.

GARDE, Ajay. Sustainable by design?: insights from US LEED-ND pilot projects. Journal of the American Planning Association, v. 75, n. 4, p. 424-440, 2009.

GHALAYINI, A. M.; NOBLE, J. S. The changing basis of performance measurement. International Journal of Operations \& Production Management, v. 16, n. 8, p. 63-80,1996.

GREENWOOD, Ted. Bridging the divide between community indicators and government performance measurement. National Civic Review, v. 97, n. 1, p. 55-59, 2008.

HAAPIO, Appu. Towards sustainable urban communities. Environmental Impact Assessment Review, v. 32, n. 1, p. 165-169, 2012.

HIREMATH, Rahul B.; Balachandra, P.; Kumar, Bimlesh; Bansode, Sheelratan S.; Murali, J.. Indicator-based urban sustainability - a review. Energy for sustainable development, v. 17, n. 6, p. 555-563, 2013.

KNACK, R. E. LEED-ND: What the skeptics say. Planning, v. 76, p. 18-21, 2010. 
KOMEILY, Ali; SRINIVASAN, Ravi S. A need for balanced approach to neighborhood sustainability assessments: A critical review and analysis. Sustainable Cities and Society, v. 18, p. 32-43, 2015.

KOMEILY, Ali; SRINIVASAN, Ravi S. What is Neighborhood Context and Why does it Matter in Sustainability Assessment?. Procedia Engineering, v. 145, p. 876-883, 2016.

KYRKOU, Dimitra; KARTHAUS, Roland. Urban sustainability standards: predetermined checklists or adaptable frameworks?. Procedia Engineering, v. 21, p. 204-211, 2011.

LUEDERITZ, C.; LANG, D. J.; VON WEHRDEN, H. A systematic review of guiding principles for sustainable urban neighborhood development. Landscape and Urban Planning, v. 118, p. 40-52, 2013.

MADANIPOUR, Ali. Roles and challenges of urban design. Journal of Urban Design, v. 11, n. 2, p. 173-193, 2006.

NEACȘU, Marius Cristian. The city image and the local public administration: a working tool in urban planning. Transylvanian Review of Administrative Sciences, v. 5, n. 27, p. 172-188, 2009.

NEELY, A. The performance measurement revolution: why now and what next? International Journal of Operations \& Production Management, v. 19, n. 2, p. 205228,1999

NEELY, A.; GREGORY, M. J.; PLATTS, K. Performance measurement system design - A literature review and research agenda. International Journal of Operations \& Production Management, v. 15, n. 4, p. 80-116, 1995.

NUDURUPATI, S. S.; BITITCI, U. S.; KUMAR, V.; CHAN, F. T. S. State of the art literature review on performance measurement. Computers \& Industrial Engineering, v. 60, n. 2, p. 279-290, 2011.

NUDURUPATI, S. S.; TEBBOUNE, S.; HARDMAN, J. Contemporary performance measurement and management (PMM) in digital economies. Production Planning \& Control, v. 27, n. 3, p. 226-235, 2016.

OLDENHUIZING, Jan; DE KRAKER, Joop; VALKERING, Pieter. Design of a quality-of-life monitor to promote learning in a multi-actor network for sustainable urban development. Journal of Cleaner Production, v. 49, p. 74-84, 2013.
REITH, András; OROVA, Melinda. Do green neighbourhood ratings cover sustainability?. Ecological Indicators, v. 48, p. 660-672, 2015.

ROHE, William M. From local to global: One hundred years of neighborhood planning. Journal of the American Planning Association, v. 75, n. 2, p. 209-230, 2009.

SHARIFI, Ayyoob; MURAYAMA, Akito. A critical review of seven selected neighborhood sustainability assessment tools. Environmental Impact Assessment Review, v. 38, p. 73-87, 2013.

SRIVASTAVA, Malini. Framework to Assess City-scale Sustainability. Procedia Engineering, v. 145, p. 1440-1447, 2016.

TALEN, Emily; ALLEN, Eliot; BOSSE, Amanda; AHMANN, Josh; KOSCHINSKY, Julia; WENTZ, Elizabeth; ANSELIN, Luc. LEED-ND as an urban metric. Landscape and Urban Planning, v. 119, p. 20-34, 2013.

TURCU, Catalina. Re-thinking sustainability indicators: local perspectives of urban sustainability. Journal of Environmental Planning and Management, v. 56, n. 5, p. 695-719, 2013.

VALMORBIDA, S. M. I.; ENSSLIN, S. R. Avaliação de Desempenho de Rankings Universitários: Revisão da Literatura e diretrizes para futuras investigações. In: Anais do XXXIX Encontro da ANPAD (EnANPAD 2015) Belo Horizonte MG. 13 a 16 de setembro de 2015. 